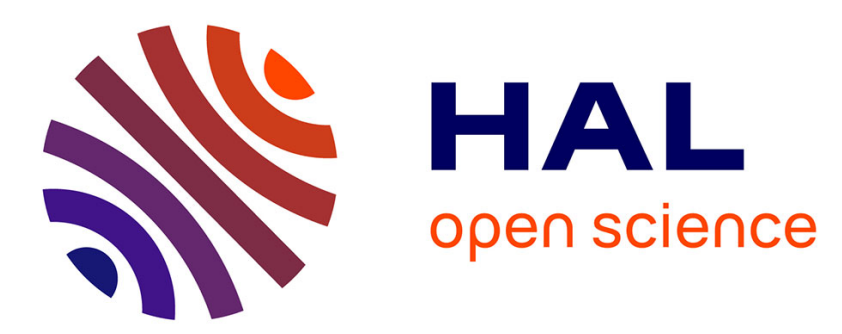

\title{
Design of a Battery-powered Emitter dedicated to the Characterization of Human Body Communications
}

\author{
R. Assila Belhadj Mefteh, R. Vauche, N. Dehaese, J. Gaubert, H. Barthelemy
}

\section{To cite this version:}

R. Assila Belhadj Mefteh, R. Vauche, N. Dehaese, J. Gaubert, H. Barthelemy. Design of a Batterypowered Emitter dedicated to the Characterization of Human Body Communications. 2020 15th Design \& Technology of Integrated Systems in Nanoscale Era (DTIS), Apr 2020, Marrakech, Morocco. pp.1-5, 10.1109/DTIS48698.2020.9081289 . hal-02561127

\section{HAL Id: hal-02561127 \\ https://hal.science/hal-02561127}

Submitted on 20 Dec 2021

HAL is a multi-disciplinary open access archive for the deposit and dissemination of scientific research documents, whether they are published or not. The documents may come from teaching and research institutions in France or abroad, or from public or private research centers.
L'archive ouverte pluridisciplinaire HAL, est destinée au dépôt et à la diffusion de documents scientifiques de niveau recherche, publiés ou non, émanant des établissements d'enseignement et de recherche français ou étrangers, des laboratoires publics ou privés. 


\title{
Design of a Battery-powered Emitter dedicated to the Characterization of Human Body Communications
}

\author{
R. Assila Belhadj Mefteh ${ }^{1}$, R. Vauche ${ }^{1}$, N. Dehaese ${ }^{1}$, J. Gaubert $^{1}$, H. Barthelemy ${ }^{1}$ \\ ${ }^{1}$ Aix-Marseille University, Univ Toulon, CNRS, IM2NP, France
}

\begin{abstract}
Human Body Communications (HBC), which has been standardized around $21 \mathrm{MHz}$, potentially offers more favourable propagation conditions than usual wireless technologies such as ZigBee, or Bluetooth. However, due their sensitivity to the close environment, the signal attenuation cannot be accurately determined using lab equipment. For this reason, a battery-powered emitter with emitted power estimation capability is fully described in this paper. The prototype implemented in a $5 \times 5 \mathrm{~cm}$ PCB is supplied with a 3.3V battery and has been built around a microcontroller (MCU). The MCU has been programmed to generate a $21 \mathrm{MHz}$ square carrier and to under-sample it at $480 \mathrm{ksps}$ using the MCU embedded Analog-to-Digital Converter (ADC). To enable the carrier power estimation with this constraint, the Goertzel light discrete Fourier transform algorithm has been implemented using particular parameters values. Finally, to overcome the bandwidth limitations of the ADC MCU pin, an external sample $\&$ hold circuit driven by the MCU have been added and optimized.
\end{abstract}

Index Terms-Goertzel algorithm, HBC, under-sampling processing, microcontroller-based emitter, power estimation

\section{INTRODUCTION}

Human Body Communications (HBC) are the third physical (PHY) layer defined by the IEEE 802.15.6 standard dedicated to Wireless Body Area Networks (WBAN) which operate close to human body. This PHY layer is primarily responsible for establishing a reliable and physical link to transmit binary data between receiver and transmitter or other equipments with a speed going up to $1.3125 \mathrm{Mbits} / \mathrm{s}$ and uses a low carrier frequency centered on $21 \mathrm{MHz}$ and a large $3 \mathrm{~dB}$ bandwidth of $5.25 \mathrm{MHz}$ [1].

On the contrary of usual standardized wireless communications such as ZigBee or Bluetooth which use air as a propagation channel [2], the HBC mark a real technological breakthrough since they propose to use the human body as a propagation medium, which potentially offers more favorable propagation conditions, better data protection and more integrated transmitters.

HBC can be associated with two types of Body Coupled Communications (BCC) where antennas are replaced by electrodes as shown in Fig. 1. The first is the galvanic coupling [3][4] where the four required electrodes are signal electrodes fixed on the human body, two on the emitter side and two on the receiver side. The signal is also differentially applied between the two emitter electrodes. The second is the capacitive coupling [3][4] which equally implies the use of four electrodes. However, two of the four required electrodes are signal electrodes attached to the human body, while the two others are ground electrodes detached from the body. Thus, a current loop, passing through the human body and the close environment such as the ground, is created and allows the signal to go from the emitter to the receiver. In this context, the propagation medium can be considered as a transmission line where the human body is the forward path and the close environment is the return path.

Since HBC were standardized, several studies [5]-[8] have been conducted to characterize the human body as a propagation channel. A comparison between measured human body path loss founded in literature is shown in Fig. 2 [9]. These measurements show that the human body path loss around $21 \mathrm{MHz}$ is distributed on a large range which is between $22 \mathrm{~dB}$ and $62 \mathrm{~dB}$. This variability can be explained in part by the different experimental conditions used to carry out the measurements such as the coupling type (capacitive or galvanic), the electrodes type, the electrodes position, and the inter-electrodes distance. However, the different equipments used to perform the measurements (balun, couplers, cables, vector network analyzer, etc.) have a higher impact on the measured path loss, especially in the case of a capacitive coupling where the path loss highly depends of the close environment. Indeed, parasitic couplings can be created by the use of main-supplied equipments via the electrical earth, or just by the use of long wires. Therefore, to make the measurement under realistic conditions, a compact batterypowered transmitter capable of estimating the emitted power and a compact battery-powered receiver capable of estimating the received power, are required to properly characterize the path loss of the human body in the context of HBC.

For this reason, this work proposes a transmitter capable of estimating the emitted power for the HBC frequency band without external measurement devices. It is based on a microcontroller and is supplied by a battery to isolate it from the main. Finally, to allow the estimation of the emitted power, the Goertzel algorithm [10], which is a light discrete Fourier transform algorithm, has been implemented using particular parameters values.

The paper is organized as follows. In section II, the architecture of the proposed transmitter is presented. In section III, the Goertzel algorithm is described and the choice of its associated parameters is detailed. Finally, measurement results are shown in section IV. 


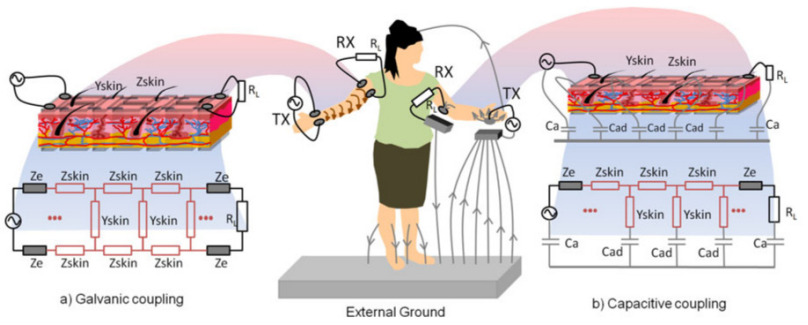

Fig. 1. The two types of body-coupled communications: a) the galvanic coupling, b) the capacitive coupling [4].

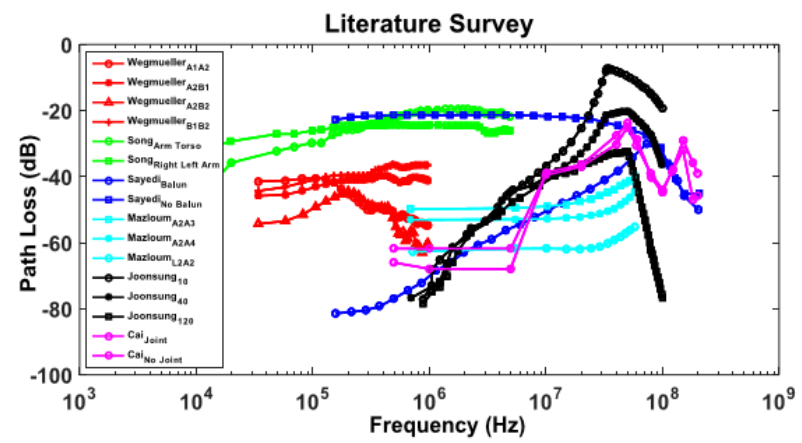

Fig. 2. Comparison between the measures of the human body path loss found in [9].

\section{DESIGN OF THE PRoposed TRANSMitTER}

\section{A. Architecture of the proposed transmitter}

The complete block diagram of the proposed transmitter is shown in the Fig. 3.a. A $21 \mathrm{MHz}$ square wave is also generated on a DSPIC microcontroller (MCU) pin and applied to the signal emitting electrode, the MCU being clocked at $84 \mathrm{MHz}$ using a $20 \mathrm{MHz}$ crystal oscillator. In addition, this signal is connected to the ADC MCU which is triggered by rising edges of a $480 \mathrm{kHz}$ signal generated by the DSPIC PWM peripheral (PWMH signal) to under-sample the $21 \mathrm{MHz}$ square wave at $480 \mathrm{ksps}$. Next, the samples are used to estimate the power level emitted in $\mathrm{dBm}$ with help of Goertzel discrete Fourier transform algorithm. Finally, the power emitted is displayed on an OLED screen.

To overcome the bandwidth limitations of the ADC MCU pin which is equal to $0.5 \mathrm{MHz}$, an external Sample \& Hold circuit $(\mathrm{S} \& \mathrm{H})$ which consists in a switch (74LVC1G3157) have been added as shown in the Fig. 3.b. This switch is controlled by a $480 \mathrm{kHz}$ signal generated by the MCU PWM peripheral (PWML signal). During the sampling phase, PWML is equal to 0 and the $21 \mathrm{MHz}$ square wave is connected to the storage capacitor $\mathrm{C}$. During the hold phase, PWML is equal to 1 and the voltage stored in $\mathrm{C}$ is connected to the ADC input which enables the analog-to-digital conversion.

To ensure a synchronization between the hold phase and the analog-to-digital conversion, the PWMH signal which triggers the ADC is shifted a quarter period from the PWML signal which control the switch as shown in the Fig. 4.

\section{B. Choice of storage capacity}

The proposed external sample and hold circuit is controlled by a $480 \mathrm{kHz}$ signal generated by the DSPIC PWML pin with a $50 \%$ duty cycle. During the sampling phase (PWML $=0$ ), the $21 \mathrm{MHz}$ square wave is also transmitted to the storage capacity $\mathrm{C}$ across the switch which can be considered as a resistor $R_{o n}$. Thus, during this phase, the circuit can be approximated by a simple RC circuit as shown in Fig. 5 [10][12].

Considering that after $5 \tau$ the capacitor is fully charged (where $\tau=R_{o n} C$ is the time constant of the circuit), the condition to have a sufficiently fast charging time is expressed as follows:

$$
5 \tau<\frac{T_{S}}{2}
$$

which leads to:

$$
C<\frac{T_{S}}{5 \times 2 \times R_{o n}}
$$

where, $T_{S}$ is the sampling period. Since the typical value of $R_{o n}$ for the used switch is $6.5 \Omega$, it also appears that $C$ must be less than $32 \mathrm{nF}$.

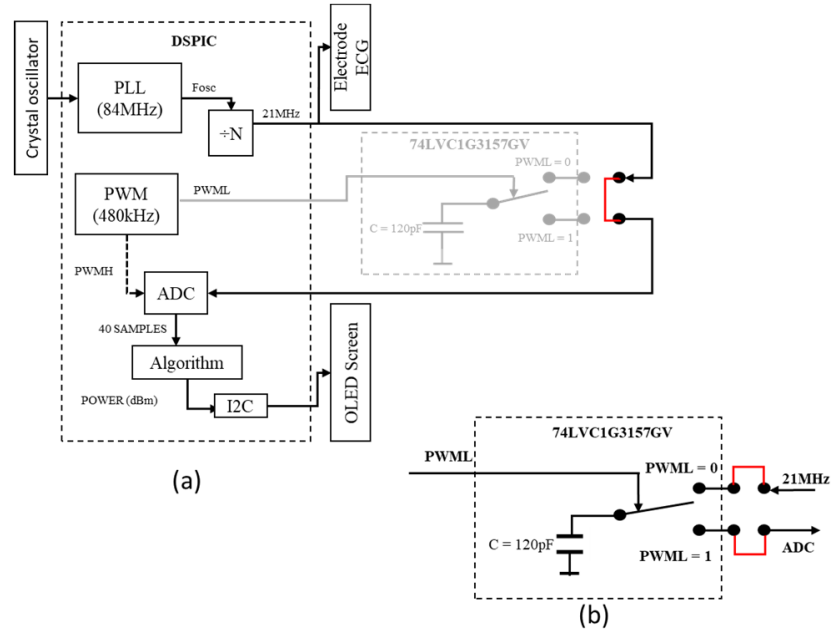

Fig. 3. Block diagram of the proposed Transmitter: without external S\&H (a) and with external $\mathrm{S} \& H(b)$.

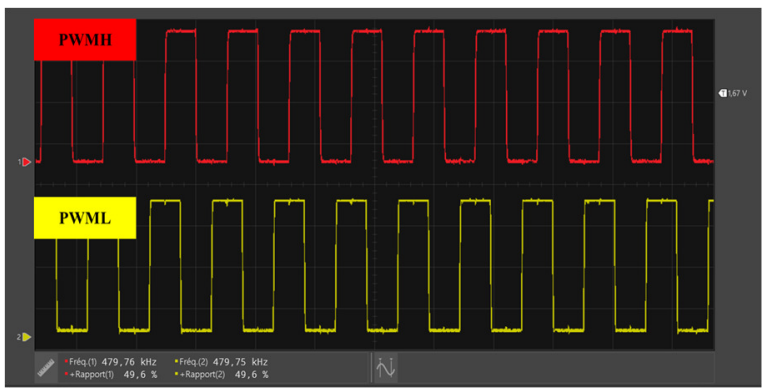

Fig. 4. 480kHz PWMH and PWML signals (1V/div and $2 \mu \mathrm{s} / \mathrm{div})$.

During the hold phase (PWML $=1)$, the stored voltage is connected to the ADC input and it can be assumed that the capacitor discharges at a constant current $I_{\text {leak }}$ which is equal to the leakage current of the ADC input as shown in Fig. 6 
( $I_{\text {leak }}=6.5 \mu \mathrm{A}$ from DSPIC datasheet). Thus, the condition to ensure that the capacitor discharge is lower than the ADC quantum $q$ is:

$$
C>\frac{T_{c} \times I_{\text {leak }}}{q}
$$

where $T_{c}$ is the time necessary for analog-to-digital conversion ( $T_{c}=0.5 \mu \mathrm{s}$ from DSPIC datasheet) and $\mathrm{q}$ is the quantum which is equal to $V_{r e f} / 2^{N}\left(V_{r e f}=3.3 \mathrm{~V}\right.$ and $N=10$ bits $)$. As result, $\mathrm{C}$ must be greater than $93 \mathrm{pF}$.

Finally, it has been found in measurement that the optimal capacitor value is $120 \mathrm{pF}$ which fulfills the conditions (2) and (3).

\section{GOERTZEL ALGORITHM}

Goertzel algorithm [10] is an algorithm used in signal processing, which performs a discrete Fourier transform of a signal $v[n]$ for a single frequency $f$. Therefore, it is proposed here to use this algorithm to estimate the power of the $21 \mathrm{MHz}$ carrier.

First, a parameter $\alpha$ is defined as follows:

$$
\alpha=\frac{2 \pi f}{F_{S}}
$$

where $f$ is the nonzero frequency of interest and $F_{S}$ is the sampling frequency, the both being chosen to ensure that $\alpha$ is not a multiple of $\pi$ which never happens if Nyquist-Shannon sampling theorem is respected. Then, the sequence $g[n]$ is computed using $N$ samples of the voltage $v[n]$ such that:

$$
\begin{gathered}
g[1]=v[1] \\
g[2]=v[2]+2 \cos \alpha \times g[1] \\
g[n]=v[n]+2 \cos \alpha \times g[n-1]-g[n-2]
\end{gathered}
$$

Finally, magnitude and phase of the signal $v[n]$ associated to the frequency $f$ can be computed as follows:

$$
\begin{gathered}
A g=\frac{\sqrt{g[N]^{2}+g[N-1]^{2}-2 g[N] g[N-1] \cos \alpha}}{N / 2} \\
\varphi g=\tan ^{-1}\left(\frac{g[N-1] \sin \alpha}{g[N]-g[N-1] \cos \alpha}\right)
\end{gathered}
$$

and the associated real and imaginary parts can be obtained using:

$$
\begin{gathered}
\operatorname{Re}\left[A g \times e^{j \varphi g}\right]=\frac{g[N]-g[N-1] \cos \alpha}{N / 2} \\
\operatorname{Im}\left[A g \times e^{j \varphi g}\right]=\frac{g[N-1] \sin \alpha}{N / 2}
\end{gathered}
$$

\section{A. Choice of sampling speed and number of samples}

The sampling frequency $F_{S}$ and the number of samples $N$ on which the Goertzel algorithm is applied must be carefully chosen. Indeed, $F_{S}$ can be expressed as a function of the frequency of interest $f$ as follows:

$$
F_{S}=\frac{p}{q} f
$$

where $p$ is an integer greater than or equal to 3 and $q$ is an integer greater than or equal to 1 to ensure the convergence of $A g$ (resp. $\varphi g$ ) to the magnitude (resp. the phase) associated to the signal $v[n]$ at the frequency $f$ in the Fourier's domain. In addition, using (12) in (4), $\alpha$ can be written as follows:

$$
\alpha=\frac{2 q}{p} \pi
$$

where $p$ and $q$ must be chosen so that $\alpha$ is not a multiple of $\pi$. In other words, $2 q / p$ must not be an integer to avoid that $\sin \alpha$ $=0$ which freezes the Goertzel algorithm in real domain $(\varphi g$ $=0)$. According to (4), this means for under-sampling condition $\left(F_{S}<2 f\right)$ that $2 f$ must not be a multiple of $F_{S}$.

Considering the proposed architecture, the Goertzel algorithm must be implemented in the DSPIC which generates the $21 \mathrm{MHz}$ square wave $(21 \mathrm{MHz}$ being the standardized carrier frequency for HBC communications) and acquires the $\mathrm{N}$ samples. Therefore, the DSPIC oscillation frequency $\left(F_{\text {osc }}\right)$ has been chosen as a multiple of $f$ as follows:

$$
f=\frac{\text { Fosc }}{4}
$$

which leads to $F_{\text {osc }}=84 \mathrm{MHz}$ since $f$ is equal to $21 \mathrm{MHz}$.

By associating (12) and (14), the following equation is obtained:

$$
\frac{F o s C}{F_{S}}=\frac{4 q}{p}
$$

A way to guarantee that $2 p / q$ is not an integer, as required by the Goertzel algorithm since $\alpha$ must not be a multiple of $\pi$, is to consider an odd number for $4 p / q$. Thus, a simple solution is to choose $p$ equals 4 and $q$ equals an odd number.

To determine $\mathrm{q}$, it is now necessary to take into account the maximum sampling frequency of the DSPIC which is here 500ksps. Therefore, the odd integer $q$ that enables to obtain the higher finite whole sampling frequency less than $500 \mathrm{kHz}$ is 175 . As result, the obtained sampling frequency is $480 \mathrm{kHz}$.

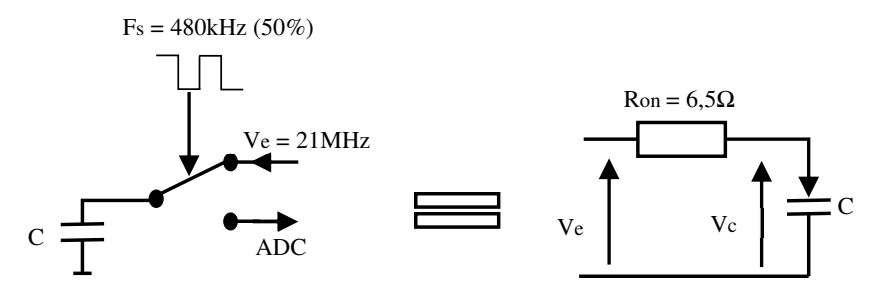

Fig. 5. Equivalent circuit of the external sample and hold circuit during the sampling phase.

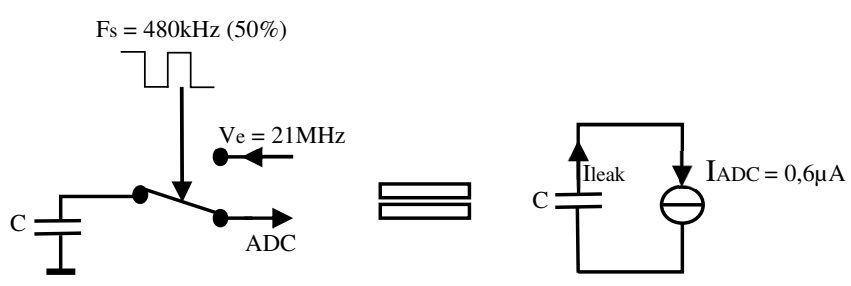

Fig. 6. Equivalent circuit of the external sample and hold circuit during the hold phase. 
Since the convergence between the Fourier series and the Goertzel algorithm is p-periodic for a noise-free signal, the algorithm must be applied for a true signal on $N$ samples with:

$$
N=r \times p
$$

where $r$ is an integer. To ensure the algorithm is stabilized, $r$ is here taken equal to 10 . As result, the number of samples obtained is 40 .

\section{B. Test of the Goertzel algorithm using MATLAB}

The Goertzel algorithm has been first implemented with the parameters indicated above $\left(f=21 \mathrm{MHz}\right.$ and $\left.\mathrm{F}_{\mathrm{S}}=480 \mathrm{kHz}\right)$ using the MATLAB software and applied on ideal $21 \mathrm{MHz}$ square and sine signals between $0 \mathrm{~V}$ and $3.3 \mathrm{~V}$ sampled at $480 \mathrm{kHz}$ as shown in the Fig. 7.

To verify the accuracy of the Goertzel algorithm with theses under-sampling conditions, the magnitude $A g$ obtained using the Goertzel algorithm are compared in Tab. I to the magnitude associated to the frequency $f$ in Fourier's domain. The latter can be obtained using the usual Fourier series and is equal to $4 \mathrm{~A} / \pi$ for a square wave, and $\mathrm{A}$ for a sine wave (with $\mathrm{A}=1.65 \mathrm{~V}$ for the both since waves are between $0 \mathrm{~V}$ and $3.3 \mathrm{~V})$. An error of $11 \%$ is obtained for the square wave due to aliasing of harmonic frequencies, which is acceptable considering the under-sampling condition. However, results perfectly match for the sine wave, but requires an additional band-pass filter to be obtained from a square wave as the one generated by the DSPIC. Finally, $\varphi g$ is not provided here but perfectly matches for the both waves when $f$ is considered.

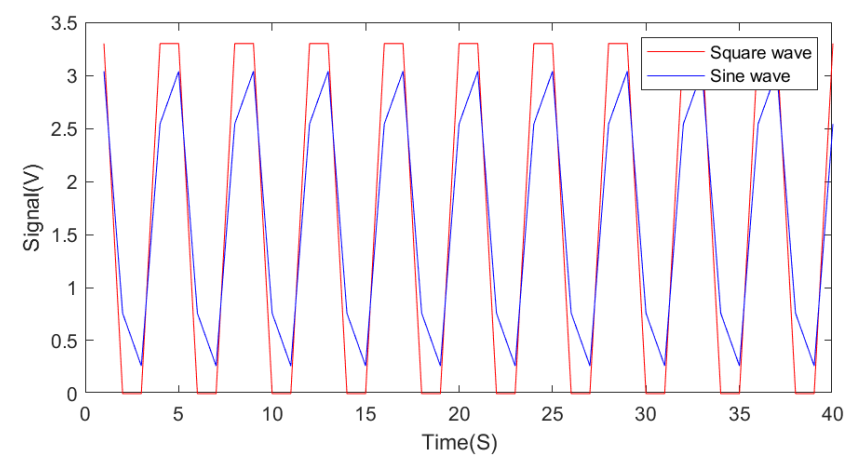

Fig. 7. $21 \mathrm{MHz}$ square and sine waves between $0 \mathrm{~V}$ and $3.3 \mathrm{~V}$ sampled at $480 \mathrm{kHz}$.

TABLE I: Magnitude comparison obtained with Goertzel algorithm and Fourier series.

\begin{tabular}{|c|c|c|c|}
\cline { 2 - 4 } \multicolumn{1}{c|}{} & $\begin{array}{c}\text { Goertzel Algorithm } \\
\text { using MATLAB (V) }\end{array}$ & $\begin{array}{c}\text { Fourier series } \\
(\mathrm{V})\end{array}$ & $\begin{array}{c}\text { Error } \\
(\%)\end{array}$ \\
\hline $\begin{array}{c}\text { Under-sampled } \\
\text { square wave }\end{array}$ & 2.33 & 2.10 & 11.0 \\
\hline $\begin{array}{c}\text { Under-sampled } \\
\text { sine wave }\end{array}$ & 1.65 & 1.65 & 0 \\
\hline
\end{tabular}

\section{MEASUREMENT RESULTS}

The proposed transmitter has been implemented in a $5 \mathrm{~cm}$ $x 5 \mathrm{~cm}$ PCB as shown in Fig. 8. It is based on a DSPIC supplied with a $3.3 \mathrm{~V}$ battery and clocked at $84 \mathrm{MHz}$ using a $20 \mathrm{MHz}$ crystal oscillator. As indicated before, the DSPIC has been programmed to generate a $21 \mathrm{MHz}$ square wave and to under-sample it at 480ksps using an external sample \& hold circuit controlled by the DSPIC too. To allow power estimation of the $21 \mathrm{MHz}$ carrier, the Goertzel discrete Fourier transform algorithm has been implemented as indicated in section III. To get an image of the emitted power, $A g$ is converted in $\mathrm{dBm}$ as shown in Fig. 8 using:

$$
P(d B m)=10 \log _{10}\left(\frac{A g^{2}}{2 \times R}\right)+30
$$

where $R$ is the fictive resistor equal to $1 \Omega$. Finally, the $21 \mathrm{MHz}$ square wave is applied to a standard $3 \mathrm{~cm}$ electrode used for electrocardiogram measurement and the ground electrode necessary for capacitive coupling consists of the PCB ground floor.

Fig. 9 shows a comparison between the values sampled with and without the external sample \& hold circuit, which should be ideally 0 or 1023 . As expected, it appears that the signal obtained using the external sample \& hold circuit is closer to the ideal under-sampled signal than the one obtained without it. However, its $\mathrm{Ag}$ value leads to an error of $24 \%$ when compared to the one of the ideal under-sampled square wave as shown in Tab. II. This difference can be explained by the bandwidth of the external sample \& circuit which is unfortunately not enough (even if better) to obtain an undersampled signal closer to the ideal under-sampled square wave since most of square harmonic frequencies are filtered. Nevertheless, the signal obtained using the external sample $\&$ hold looks like the ideal under-sampled sine wave and its $A g$ value leads to an error of only $6.7 \%$ when compared to the one of this sine wave as shown in Tab. III.

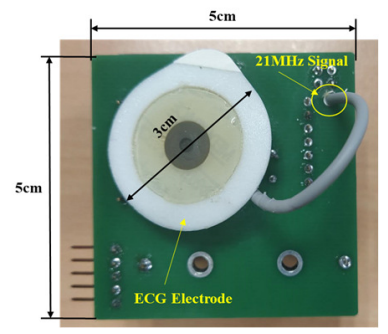

(a)

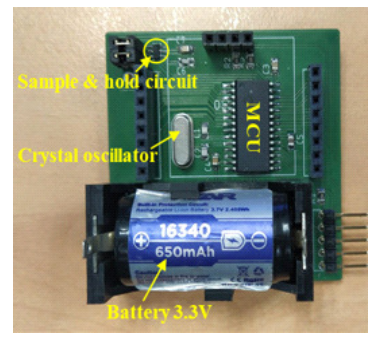

(b)

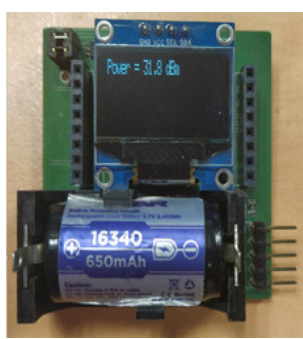

(c)
Fig. 8. Photographs of the designed transmitter. 


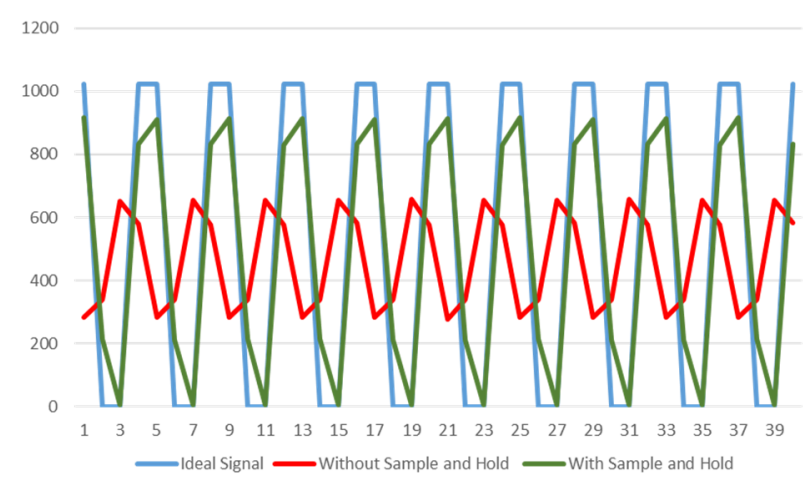

Fig. 9. Comparison of the forty 10bit-samples when the $21 \mathrm{MHz}$ square wave is under-sampled at $480 \mathrm{kHz}$.

TABLE II: Comparison of the $A g$ value obtained with the Goertzel algorithm using the designed prototype and an ideal under-sampled square wave.

\begin{tabular}{|c|c|c|c|}
\cline { 2 - 4 } \multicolumn{1}{c|}{} & $\begin{array}{c}\text { Without } \\
\text { external S\&H }\end{array}$ & $\begin{array}{c}\text { With } \\
\text { external S\&H }\end{array}$ & $\begin{array}{c}\text { Ideal under-sampled } \\
\text { square wave } \\
\text { (MATLAB) }\end{array}$ \\
\hline$A g(\mathrm{~V})$ & 0.84 & 1.76 & 2.33 \\
\hline Error $(\%)$ & $64 \%$ & $24 \%$ & 0 \\
\hline
\end{tabular}

TABLE III: Comparison of the $A g$ value obtained with the Goertzel algorithm using the designed prototype and an ideal under-sampled sine wave.

\begin{tabular}{|c|c|c|c|}
\cline { 2 - 4 } \multicolumn{1}{c|}{} & $\begin{array}{c}\text { Without } \\
\text { external S\&H }\end{array}$ & $\begin{array}{c}\text { With } \\
\text { external S\&H }\end{array}$ & $\begin{array}{c}\text { Ideal under-sampled } \\
\text { sine wave } \\
\text { (MATLAB) }\end{array}$ \\
\hline$A g(\mathrm{~V})$ & 0.84 & 1.76 & 1.65 \\
\hline Error $(\%)$ & $49 \%$ & $6.7 \%$ & 0 \\
\hline
\end{tabular}

\section{CONCLUSION}

Human Body Communications (HBC), which has been standardized around $21 \mathrm{MHz}$, potentially offers more favourable propagation conditions than usual wireless technologies such as ZigBee, or Bluetooth. However, due their sensitivity to the close environment, the signal attenuation cannot be accurately determined using lab equipment. Therefore, to make the measurement under realistic conditions, a battery-powered emitter with emitted power estimation capability has been fully described in this paper. The prototype implemented in a $5 \times 5 \mathrm{~cm}$ PCB is supplied with a $3.3 \mathrm{~V}$ battery and has been built around a MCU. The MCU has been programmed to generate a $21 \mathrm{MHz}$ square carrier and to under-sample it at 480ksps using the MCU embedded Analog-to-Digital Converter (ADC). To enable the carrier power estimation with this constraint, the Goertzel light discrete Fourier transform algorithm has been implemented using particular parameters values. Moreover, to overcome the bandwidth limitations of the ADC MCU pin, an external sample \& hold circuit driven by the MCU have been added and optimized. However, a filter could be added in the designed transmitter to completely filter harmonic frequencies of the square wave and also to improve power estimation using Goertzel Algorithm when an undersampling is considered as in this work. Finally, to properly characterize the path loss of the human body, a compact receiver capable of estimating the received power has now to be designed.

\section{REFERENCES}

[1] IEEE Com.Soc., "IEEE standard for Local and metropolitan area network - Part 15.6: Wirless Body Area Networks," IEEE std 802.15.6-2012, pp. 1-271, 29 Feb 2012.

[2] C. E. Shannon, "A Mathematical Theory of Communication," The Bell System Technical Journal, vol. 27, pp. 379-423, 623-656, July, Oct.1948.

[3] M. A. Callejón, D. Naranjo-Hernandez, J. Reina-Tosina and L. M. Roa, "Distributed Circuit Modeling of Galvanic and Capacitive Coupling for Intrabody Communication," in IEEE Transactions on Biomedical Engineering, vol. 59, no. 11, pp. 3263-3269, Nov. 2012.

[4] M. Seyedi, B. Kibret, D. T. H. Lai and M. Faulkner, "An empirical comparison of limb joint effects on capacitive and galvanic coupled intra-body communications," 2013 IEEE Eighth International Conference on Intelligent Sensors, Sensor Networks and Information Processing, Melbourne, VIC, 2013, pp. 213-218

[5] M. S. Wegmueller, M. Oberle, N. Felber, N. Kuster and W. Fichtner, "Signal Transmission by Galvanic Coupling Through the Human Body," in IEEE Transactions on Instrumentation and Measurement, vol. 59, no. 4, pp. 963-969, April 2010.

[6] J. Bae, H. Cho, K. Song, H. Lee and H. Yoo, "The Signal Transmission Mechanism on the Surface of Human Body for Body Channel Communication," in IEEE Transactions on Microwave Theory and Techniques, vol. 60, no. 3, pp. 582-593, March 2012.

[7] M. H. Sayedi and D. Lai, "A novel intrabody communication transceiver for biomedical applications," Ph.D. dissertation, College Eng. Sci., Victoria Univ., Footscray, VIC, Australia, 2014.

[8] Z.Cai, "Impulse radio intrabody communication system for wireless body area networks," Ph.D. dissertation, College Eng. Sci., Victoria Univ., Footscray, VIC, Australia, 2015.

[9] S. Maity, K. Mojabe and S. Sen, "Characterization of Human Body Forward Path Loss and Variability Effects in Voltage-Mode HBC," in IEEE Microwave and Wireless Components Letters, vol. 28, no. 3, pp. 266-268, March 2018.

[10] G. Goertzel, « An Algorithm for the Evaluation of Finite Trigonometric Series », The American Mathematical Monthly, vol. 65, no. 1, pp. 34$35,1958$.

[11] G. Xu and J. Yuan, "Accurate sample-and-hold circuit model," in Electronics Letters, vol. 41, no. 9, pp. 520-522, 28 April 2005.

[12] G. K. De Teyou, H. Petit, P. Loumeau and H. Fakhoury, "Statistical analysis of harmonic distortion in a differential bootstrapped sample and hold circuit," 2014 10th Conference on Ph.D. Research in Microelectronics and Electronics (PRIME), Grenoble, 2014, pp. 1-4. 